
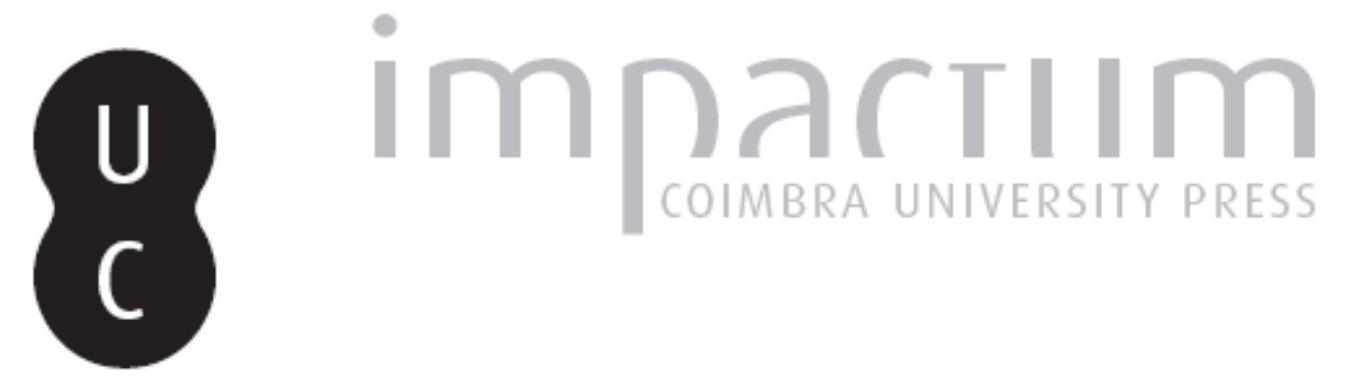

\title{
Análise química de um lote de escórias do Castelo Velho de Alcoutim e o projecto de Cartografia Geoquímica do país
}

\author{
Autor(es): $\quad$ Morgado, Paulo
}

Publicado por: Faculdade de Letras da Universidade de Coimbra

URL persistente:

URI:http://hdl.handle.net/10316.2/36406

DOI:

DOI:http://dx.doi.org/10.14195/1647-8657_51_6

Accessed : $\quad$ 26-Apr-2023 16:35:22

A navegação consulta e descarregamento dos títulos inseridos nas Bibliotecas Digitais UC Digitalis, UC Pombalina e UC Impactum, pressupõem a aceitação plena e sem reservas dos Termos e Condições de Uso destas Bibliotecas Digitais, disponíveis em https://digitalis.uc.pt/pt-pt/termos.

Conforme exposto nos referidos Termos e Condições de Uso, o descarregamento de títulos de acesso restrito requer uma licença válida de autorização devendo o utilizador aceder ao(s) documento(s) a partir de um endereço de IP da instituição detentora da supramencionada licença.

Ao utilizador é apenas permitido o descarregamento para uso pessoal, pelo que o emprego do(s) título(s) descarregado(s) para outro fim, designadamente comercial, carece de autorização do respetivo autor ou editor da obra.

Na medida em que todas as obras da UC Digitalis se encontram protegidas pelo Código do Direito de Autor e Direitos Conexos e demais legislação aplicável, toda a cópia, parcial ou total, deste documento, nos casos em que é legalmente admitida, deverá conter ou fazer-se acompanhar por este aviso.

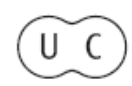




\section{CONIMBRIGA}

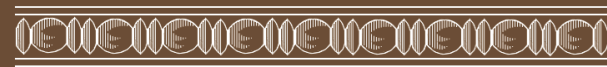

TIIDNIDN

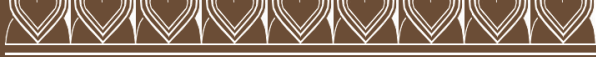

INSTITUTO DE ARQUEOLOGIA

VOLUME LI • 2012

FACULDADE DE LETRAS UNIVERSIDADE DE COIMBRA 


\title{
Paulo Morgado
}

Engenheiro Geólogo, Universidade de Aveiro

pmorgado@ua.pt

\author{
ANÁLISE QUÍMICA DE UM LOTE DE ESCÓRIAS DO CASTELO \\ VELHO DE ALCOUTIM E O PROJECTO DE CARTOGRAFIA \\ GEOQUÍMICA DO PAÍS \\ “Conimbriga" LI (2012) p. 151-161
}

Resumo: A intervenção arqueológica realizada no Castelo Velho de Alcoutim permitiu identificar uma série de estruturas e materiais associadas à actividade metalúrgica. Análises químicas efectuadas sobre algumas amostras de escórias e fragmentos de rocha identificados como matéria-prima, permitiram uma leitura de possível associação a anomalias geoquímicas, identificadas no projecto de cartografia geoquímica global do país, realizado com amostragem de baixa densidade. Elevados teores de alguns elementos químicos, identificados analiticamente, puderam ser associados aos teores médios classificados segundo a Zona Estrutural e segundo a Litologia.

Palavras Chave: Cartografia geoquímica; escórias; mineração; metalurgia.

ABSTRACT: The archaeological intervention performed in Castelo Velho de Alcoutim (the Old Castle of Alcoutim) was able to identify a number of structures and materials associated with metallurgical activity. Chemical analysis on some slag samples and rock fragments, identified as raw materials, allowed a reading of possible association to geochemical anomalies identified in the country's global geochemical mapping project, carried out with low density samples. High levels of some chemical elements, analytically

Conimbriga, 51 (2012) 151-161 
identified, where associated to average levels classified according to the Structural Zone and according to Lithology.

KEYWORDS: Geochemical mapping; slags; ore; metallurgy. 


\section{ANÁLISE QUÍMICA DE UM LOTE DE ESCÓRIAS \\ DO CASTELO VELHO DE ALCOUTIM E O PROJECTO DE CARTOGRAFIA GEOQUÍMICA DO PAÍS}

\section{Introdução}

Por altura da intervenção arqueológica no Castelo Velho de Alcoutim $^{1}$ foi apresentado o trabalho de elaboração da Cartografia Geoquímica do país (FERREIRA, 2000), no âmbito do projecto IGCP 259 (International Geological Correlation Programme, project ${ }^{\circ} 259$ ).

Em 1988 havia sido criado um programa internacional intitulado "International Geochemical Mapping" (IGM), com o propósito de discutir e estabelecer os melhores critérios que permitissem obter um conjunto universal de dados quantitativos de qualidade com os quais seria possível a elaboração de um atlas geoquímico do globo (DARNLEY et al., 1995). O primeiro estádio do IGM foi o projecto IGCP 259, que se definiu com o objectivo de rever, identificar problemas e discutir soluções quanto às metodologias usadas na produção de mapas geoquímicos regionais e nacionais para cada país.

O projecto $\mathrm{n}^{\circ} 259$ do IGCP teve em Portugal um grupo de trabalho que dinamizou a sua aplicação no país, formado no âmbito do ex-INIC (PINTO, comun. pess.). Vários investigadores dos comités técnicos daquele projecto estiveram reunidos numa mesa-redonda realizada durante a VII Semana de Geoquímica na qual se discutiram alguns aspectos da sua aplicação a Portugal (ALVES et al., 1991). Os objectivos principais foram a criação de uma base de dados geoquímicos de Portugal Continental referente a 32 elementos químicos, com base numa rede de amostragem de baixa densidade de sedimentos de corrente, a produção de mapas geoquímicos à pequena escala ( 1/3 000 000), assim

${ }^{1}$ Responsabilidade científica da Professora Doutora Helena Catarino. 
como contribuir para a compreensão da variação geoquímica dos elementos cartografados, aumento do conhecimento da geoquímica desses elementos em ambiente superficial, definindo valores padrão para as diferentes regiões do país assim como para as diferentes zonas geoestruturais e litologias (Fig.1). Este trabalho surge como uma tese de doutoramento realizada no Departamento de Geociências da Universidade de Aveiro (FERREIRA, 2000).

Com a publicação desta cartografia geoquímica do país, surgiu a oportunidade e possibilidade de realizar um estudo, nos anos de 2003 e 2004, com a mesma metodologia, sobre as escórias provenientes de contextos de fundição identificados no Castelo Velho de Alcoutim e verificar a possibilidade de associação com os teores médios das litologias existentes na área geográfica envolvente. É precisamente o estudo dos resultados dessas análises, sobre um lote de nove amostras, realizadas no laboratório comercial "ACME Analytical Laboratories Ltd, de Vancouver, Canadá”, que agora se apresenta.

\section{Análise química}

O estudo das tecnologias, em particular dos processos de pirometalurgia, pode fornecer indicações importantes sobre o estado de desenvolvimento das comunidades onde se dominava a técnica que permitia transformar pelo fogo a rocha no metal (SILVA, 2002). Efectivamente o estudo sobre os materiais utilizados nos processos metalúrgicos, desde a fonte da matéria-prima, passando pelos produtos obtidos sequencialmente ao longo do processo até aos produtos finais, é susceptível de fornecer dados que, indirectamente, podem indicar os métodos de trabalho aplicados, podendo estes ser indiciadores do referido estado de desenvolvimento dos processos.

É neste sentido que se seleccionou um lote de amostras, constituído por 8 amostras de escória, e uma amostra de material geológico, que estava associado a uma das escórias (amostras $n^{\circ} 9$ e 7 respectivamente), provenientes da intervenção arqueológica no Castelo Velho de Alcoutim, para serem alvo de análise química ${ }^{2}$.

2 Trabalho realizado em colaboração com o Dr. António Coelho da Rocha e Professor Doutor Serrano Pinto, do Departamento de Geociências da Universidade de Aveiro.

Conimbriga, 51 (2012) 151-161 
As nove amostras foram submetidas a tratamento físico no Departamento de Geociências da Universidade de Aveiro e análise química no laboratório comercial "ACME Analytical Laboratories Ltd, de Vancouver, Canadá.

No estudo fez-se somente a caracterização química das amostras, pela determinação dos teores de elementos químicos. Para a sua melhor caracterização, estão previstos estudos complementares como a determinação das fases minerais presentes (difracção de RX), ou a realização de superfícies polidas onde se possa efectuar a microanálise da morfologia dos minerais (microscopia electrónica de varrimento - MEV).

As amostras foram identificadas associando-as ao seu local de proveniência na escavação do Castelo Velho de Alcoutim como a seguir se apresenta:

\title{
Amostra 1
}

Escória. Campanha 10/2000 - Corte 2 - Q E16 - nível 3 -29/09/2000

\section{Amostra 2}

Escória. Campanha 12/2003 - Corte 2 - Q C18 - nível 6b $20 / 08 / 2003$

\section{Amostra 3}

Escória. Campanha 12/2003 - Corte 2 - Q B/C18 - nível 6b

\section{Amostra 4}

Escória. Campanha 12/2003 - Corte 2 - Q B/C18 - nível 6b $19 / 08 / 2003$

\section{Amostra 5}

Escória. Campanha 9/1999 - Corte 2 - Q F17 - nível 1b - 07/09/1999

\section{Amostra 6}

Escória. Campanha 9/1999 - Corte 2 - Q D18 - nível 1b - 20/08/1999

\author{
Amostra 7 \\ Escória. Campanha 12/2003 - Corte 2 - Q C18 - nível 6b - \\ 20/08/2003
}




\section{Amostra 8}

Escória. Campanha 11/2002 - Corte 2 - Q B18 - nível 5 - 04/09/2002

\section{Amostra 9}

Material geológico associado à escória $\mathrm{n}^{\mathrm{0}} 7$ (Corte 2 - Q. C18 nível 6b)

O tratamento laboratorial foi realizado segundo as orientações do projecto IGCP 259. Efectuou-se um ataque físico sobre as amostras, de modo a obter a fracção granulométrica para a análise química. As amostras foram cuidadosamente lavadas, e desagregadas num almofariz ou, quando necessário, recorrendo a um moinho de maxilas. De seguida foram seleccionadas as partes da amostra a analisar. A moagem fina fez-se recorrendo a um moinho de ágata. Este material foi por sua vez passado por uma bateria de crivos de modo a obter a fracção granulométrica a usar, que de acordo com os resultados de FERREIRA (1993) foi utilizada a fracção $<188 \mu \mathrm{m}$ ( $<80$ mesh).

A amostra assim obtida foi homogeneizada sobre uma folha de papel, onde se recolheu cerca de $2 \mathrm{~g}$ para análise laboratorial. O restante material foi guardado em contentores de plástico de $20 \mathrm{ml}$, devidamente identificados, funcionando como reserva para posteriores estudos.

O método analítico seleccionado para análise foi a Espectrometria Óptica de Emissão com plasma de indução acoplado, vulgarmente designado por Espectrometria ICP (Inductively Coupled Plasma), o que permitiu fazer a análise simultânea de trinta e seis elementos químicos por cada amostra, que se apresentam na tabela a seguir.

\begin{tabular}{|c|c|c|c|c|c|c|c|c|c|c|c|}
\hline $\begin{array}{c}\text { Ele- } \\
\text { mento }\end{array}$ & $\begin{array}{c}\text { Uni- } \\
\text { dade }\end{array}$ & $\begin{array}{c}\text { Limite } \\
\text { detecção }\end{array}$ & 1 & 2 & 3 & 4 & 5 & 6 & 7 & 8 & 9 \\
\hline $\mathrm{Ag}$ & $(\mathrm{ppm})$ & 0,1 & $<.1$ & $<.1$ & 3,5 & 0,5 & 5,4 & $<.1$ & 0,2 & 0,2 & 0,1 \\
$\mathrm{Al}$ & $(\%)$ & 0,01 & 0,68 & 0,69 & 1,61 & 1,18 & 1,7 & 1,72 & 1,56 & 1,32 & 3,57 \\
$\mathrm{As}$ & $(\mathrm{ppm})$ & 0,5 & 192 & 16,8 & 26,5 & 32,1 & 29,3 & 75,4 & 8,7 & 51,5 & 4,1 \\
$\mathrm{Au}$ & $(\mathrm{ppb})$ & 0,5 & 7,1 & 17,6 & 139,3 & 21,4 & 35,9 & 22,3 & 7,1 & 10,8 & 6 \\
$\mathrm{~B}$ & $(\mathrm{ppm})$ & 1 & 5 & 6 & 15 & 8 & 11 & 18 & 11 & 8 & 4 \\
$\mathrm{Ba}$ & $(\mathrm{ppm})$ & 1 & 86 & 43 & 152 & 64 & 213 & 194 & 101 & 90 & 47 \\
$\mathrm{Bi}$ & $(\mathrm{ppm})$ & 0,1 & $<.1$ & $<.1$ & $<.1$ & $<.1$ & $<.1$ & $<.1$ & $<.1$ & $<.1$ & 0,2 \\
$\mathrm{Ca}$ & $(\%)$ & 0,01 & 0,24 & 0,22 & 0,48 & 0,36 & 0,53 & 0,88 & 0,93 & 0,39 & 0,21 \\
$\mathrm{Cd}$ & $(\mathrm{ppm})$ & 0,1 & $<.1$ & $<.1$ & $<.1$ & $<.1$ & $<.1$ & $<.1$ & 0,1 & $<.1$ & $<.1$ \\
\hline
\end{tabular}

Conimbriga, 51 (2012) 151-161 


\begin{tabular}{|c|c|c|c|c|c|c|c|c|c|c|c|}
\hline $\begin{array}{c}\text { Ele- } \\
\text { mento }\end{array}$ & $\begin{array}{c}\text { Uni- } \\
\text { dade }\end{array}$ & $\begin{array}{c}\text { Limite } \\
\text { detecção }\end{array}$ & 1 & 2 & 3 & 4 & 5 & 6 & 7 & 8 & 9 \\
\hline $\mathrm{Co}$ & $(\mathrm{ppm})$ & 0,1 & 59,7 & 17,5 & 26,2 & 24,2 & 34,3 & 31,1 & 9,6 & 65,1 & 10,2 \\
$\mathrm{Cr}$ & $(\mathrm{ppm})$ & 1 & 11,1 & 9,1 & 23,8 & 13,9 & 23,3 & 17,8 & 16,1 & 15,2 & 16,1 \\
$\mathrm{Cu}$ & $(\mathrm{ppm})$ & 0,1 & 1517,6 & 51,3 & 117 & 134 & 1046 & 317,8 & 43,1 & 670,9 & 16,2 \\
$\mathrm{Fe}$ & $(\%)$ & 0,01 & $>40$ & 9,11 & 25,61 & 23,38 & $>40$ & 32,75 & 26,43 & 32,76 & 2,72 \\
$\mathrm{Ga}$ & $(\mathrm{ppm})$ & 1 & 6 & 3 & 7 & 6 & 11 & 5 & 5 & 5 & 11 \\
$\mathrm{Hg}$ & $(\mathrm{ppm})$ & 0,01 & 0,01 & 0,01 & $<.01$ & 0,01 & 0,03 & 0,01 & 0,01 & 0,01 & $<.01$ \\
$\mathrm{~K}$ & $(\%)$ & 0,01 & 0,28 & 0,2 & 0,67 & 0,25 & 0,79 & 1,25 & 0,43 & 0,37 & 0,26 \\
$\mathrm{La}$ & $(\mathrm{ppm})$ & 1 & 4 & 6 & 13 & 7 & 13 & 15 & 9 & 8 & 19 \\
$\mathrm{Mg}$ & $(\%)$ & 0,01 & 0,19 & 0,11 & 0,3 & 0,24 & 0,34 & 0,43 & 0,44 & 0,23 & 0,4 \\
$\mathrm{Mn}$ & $(\mathrm{ppm})$ & 1 & 802 & 1437 & 5087 & 3635 & 3706 & 6996 & 3470 & 3284 & 629 \\
$\mathrm{Mo}$ & $(\mathrm{ppm})$ & 0,1 & 16,2 & 1,3 & 3,3 & 3,4 & 5,2 & 3,5 & 2,4 & 3,7 & 0,2 \\
$\mathrm{Na}$ & $(\%)$ & 0,001 & 0,106 & 0,025 & 0,097 & 0,047 & 0,134 & 0,13 & 0,179 & 0,085 & 0,052 \\
$\mathrm{Ni}$ & $(\mathrm{ppm})$ & 0,1 & 139,8 & 31,4 & 38,4 & 41,2 & 28,7 & 58,9 & 17,9 & 68,4 & 18,3 \\
$\mathrm{P}$ & $(\%)$ & 0,001 & 0,055 & 0,045 & 0,079 & 0,091 & 0,216 & 0,161 & 0,106 & 0,073 & 0,149 \\
$\mathrm{~Pb}$ & $(\mathrm{ppm})$ & 0,1 & 0,6 & 0,7 & 0,7 & 3,2 & 13,1 & 10,2 & 2,1 & 2,3 & 5,3 \\
$\mathrm{~S}$ & $\%$ & 0,05 & $<.05$ & $<.05$ & $<.05$ & $<.05$ & $<.05$ & $<.05$ & $<.05$ & $<.05$ & $<.05$ \\
$\mathrm{Sb}$ & $(\mathrm{ppm})$ & 0,1 & 0,7 & 1 & 1 & 0,8 & 1,2 & 5,1 & 0,4 & 1 & 0,3 \\
$\mathrm{Sc}$ & $(\mathrm{ppm})$ & 0,1 & 1,7 & 1,9 & 4,8 & 2,9 & 4,5 & 4,2 & 3,1 & 2,9 & 5,1 \\
$\mathrm{Se}$ & $(\mathrm{ppm})$ & 0,5 & $<.5$ & $<.5$ & $<.5$ & $<.5$ & 0,8 & $<.5$ & $<.5$ & $<.5$ & $<.5$ \\
$\mathrm{Sr}$ & $(\mathrm{ppm})$ & 1 & 31 & 19 & 64 & 36 & 79 & 99 & 57 & 40 & 34 \\
$\mathrm{Th}$ & $(\mathrm{ppm})$ & 0,1 & 1,3 & 1,3 & 3,4 & 1,9 & 4,5 & 4,2 & 3 & 2,8 & 4,3 \\
$\mathrm{Ti}$ & $(\%)$ & 0,001 & 0,042 & 0,029 & 0,067 & 0,048 & 0,085 & 0,063 & 0,062 & 0,058 & 0,004 \\
$\mathrm{Tl}$ & $(\mathrm{ppm})$ & 0,1 & $<.1$ & $<.1$ & $<.1$ & $<.1$ & $<.1$ & $<.1$ & $<.1$ & $<.1$ & 0,1 \\
$\mathrm{U}$ & $(\mathrm{ppm})$ & 0,1 & 0,4 & 0,2 & 0,7 & 0,4 & 1,5 & 0,8 & 0,6 & 0,5 & 0,2 \\
$\mathrm{~V}$ & $(\mathrm{ppm})$ & 2 & 19 & 16 & 37 & 27 & 40 & 26 & 28 & 26 & 33 \\
$\mathrm{~W}$ & $(\mathrm{ppm})$ & 0,1 & 0,2 & 0,3 & 0,4 & 3,3 & 2,1 & 2,5 & 3,6 & 0,9 & $<.1$ \\
$\mathrm{Zn}$ & $(\mathrm{ppm})$ & 1 & 3 & 4 & 5 & 12 & 14 & 6 & 8 & 7 & 21 \\
\hline
\end{tabular}

$<-$ Valor inferior ao limite de detecção ppm - Parte por milhão ppb - Parte por bilhão

As unidades ppm e ppb utilizam-se quando os valores das concentrações são muito pequenos e têm a seguinte correspondência: $1 \%=10000 \mathrm{ppm}$ e $1 \%=10000000 \mathrm{ppb}$

Da análise realizada, verifica-se que todas as amostras estudadas apresentam algum enriquecimento em cobre, o que poderá estar associado a tratamento inicial de minerais ricos neste elemento como os sul-

Conimbriga, 51 (2012) 151-161 
furetos de ferro e cobre (calcopirite), presentes em significativa abundância na Zona Faixa Piritosa, onde a região de Alcoutim se implanta.

Em relação ao conteúdo em ferro, excetuando duas amostras $(2 \mathrm{e}$ 9) todas as outras têm elevados teores pelo que podem ser associadas à metalurgia do ferro. As amostras com teores acima da capacidade de quantificação do equipamento, acima dos 40\% (amostras 1 e 5), corresponderão a amostras de forja.

Em relação ao ouro, as amostras possuem teores significativos tendo em conta que os valores médios dos teores de ouro das rochas da região que são de 2 a 5 ppb, estando, no entanto, os valores médios das minas da Faixa Piritosa entre 2 a 4 ppm. No entanto, os teores presentes nestas amostras não são suscetíveis de serem tratados com os métodos de concentração da época.

O conteúdo em prata presente nas amostras está muito abaixo dos valores médios dos minerais da Faixa Piritosa, na ordem dos 30 a 40 ppm.

Em conclusão podemos considerar que os mapas de distribuição espacial dos teores dos elementos químicos produzidos para o País (Portugal continental), permitem reconhecer os locais ou zonas onde existem anomalias de altos teores para cada um dos elementos estudados e assim aferir da possibilidade de existirem formações geológicas com possibilidade de extracção de determinado metal a usar nos processos metalúrgicos.

A metodologia de determinação dos teores dos elementos químicos utilizada no projecto IGCP 259, aplicada aos materiais provenientes de sítios arqueológicos, permite a obtenção e identificação do seu registo químico e, por este método, a associação a um possível processo metalúrgico para obtenção de um determinado metal. 


\section{BIBLIOGRAFIA}

Alves, C. A. M.; Fonseca, E. C. \& Pinto, M. C. S., (1991) - VIII Semana de Geoquímica - Nota introdutória. Geociências, Revista da Universidade de Aveiro.

BJöRKLund, Alf (1992) - Proposals for future global geochemical sampling for presentation at IGCP 259.

Darnley, A. G.; Buörklund, A.; Bølviken, B.; Gustavsson, N.; Koval, P.V.; Plant, J.A.; Steenfelt, A.; Tauchid, M. \& Xie XuejIng, (1995). A Global Geochemical Database For Environmental And Resource Management. Recommendations for international geochemical mapping. Final report of IGCP project 259. UNESCO Publishing.

Ferreira, António M. P. J., (1993). Uso de sedimentos de corrente (amostragem de baixa densidade) na caracterização geoquímica das bacias dos rios Vouga e Mira. Implicações em Cartografia Geoquímica Regional. Dissertação de Mestrado. Universidade de Aveiro.

Ferreira, António M. P. J., (2000). Dados geoquímicos de base de sedimentos fluviais de amostragem de baixa densidade de Portugal Continental: Estudo de factores de variação regional. Dissertação de Doutoramento. Departamento de Geociências da Universidade de Aveiro.

Morgado, Paulo J. M, Catarino, Helena M. G. e Rocha, António C., (2004) Material geológico, escórias e fragmentos de bala de canhão provenientes do Castelo Velho de Alcoutim, Relatório preliminar da análise de escórias, Aveiro (policopiado).

Morgado, Paulo, Pereira, Vitória \& Pinto, Manuel S (2001) - "Chromium in Portuguese Soils Surrounding Electroplating Facilities", Environmental Geochemistry and Health 1-4, Kluwer Academic Publishers. Netherlands.

Morgado, Paulo. J. C., (1997) - Caracterização geoquímica e cartografia regional de sedimentos de corrente do Sul de Portugal. Implicações em Cartografia Geoquímica Regional. Dissertação de Mestrado. Departamento de Geociências, Universidade de Aveiro.

Péres Macias, Juan. A., (1999) - Cerro de Salomón y la Mineria Hispanomusulmna en Garb-Al-Andalus. Arqueologia Medieval 61999 19-37.

Péres Macias, Juan. A., Delgado Dominguez, A. (2012) - Ingeniería minera antigua y medieval en el suroeste Ibérico. Boletín Geológico y Minero 122.

Conimbriga, 51 (2012) 151-161 
GeOQuímicA de PORTUGAL cartografia regional 2000

(<80\#, Aqua Regia, ICP-AES; krigagem)

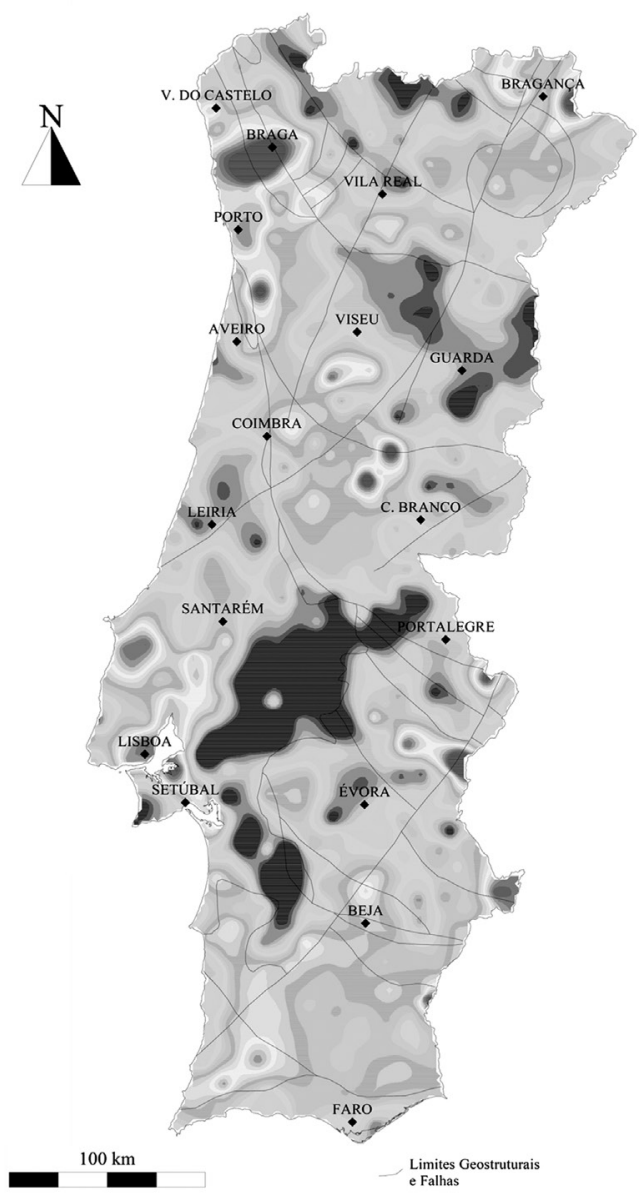

\section{$\mathrm{Cu}$}

Sedimentos

de Corrente

$\mathrm{N}=653$

percentil ppm

$\operatorname{Max}$

98

\begin{tabular}{l}
817 \\
126 \\
107 \\
91 \\
78 \\
66 \\
56 \\
\hline
\end{tabular}

$90 \quad 48$

41
35
75

75

50

25

\begin{tabular}{c|c} 
& 10 \\
& 8 \\
10 & \\
\hline & \\
\hline Min &
\end{tabular}
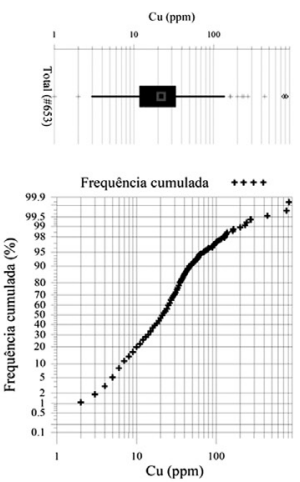

Teores em Sedimentos de Corrente de Portugal (ppm)

\begin{tabular}{|ccccccccc|}
\hline Min & p25 & GM & Mdn & Média & p75 & p98 & Max & C\% \\
1 & 12 & $\mathbf{2 0}$ & $\mathbf{2 2}$ & $\mathbf{3 0}$ & 33 & 126 & 817 & 175.0 \\
Acetato de & Amónio: & & & & & & & \\
0.10 & 0.52 & $\mathbf{1 . 1 7}$ & $\mathbf{1 . 1 2}$ & $\mathbf{4 . 6 7}$ & 2.49 & 25.93 & 577.10 & 617.1 \\
\hline
\end{tabular}

Fig.1 - Exemplo dos mapas de distribuição de teores produzidos no projecto IGCP 259. Caso do mapa para o elemento químico cobre, determinado em sedimentos fluviais e respectiva tabela de teores. 

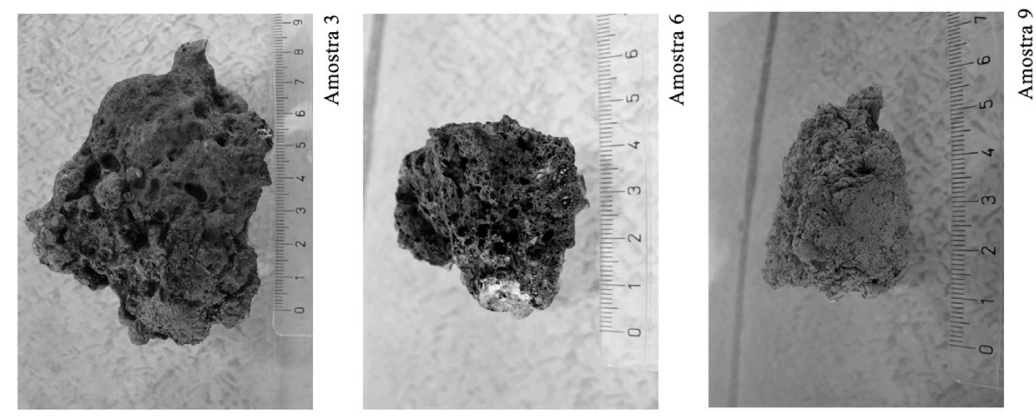

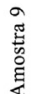
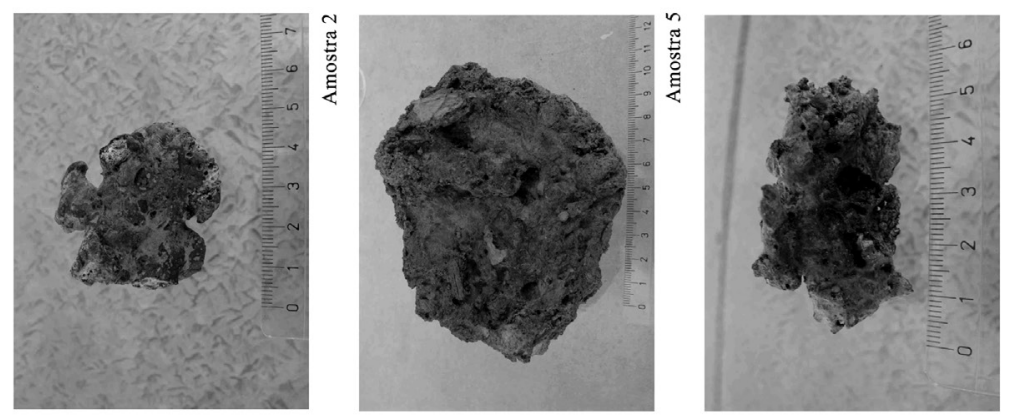

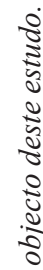

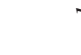

